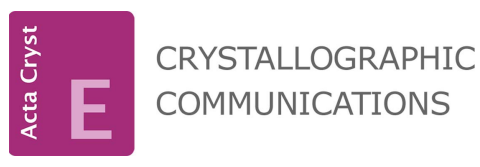

ISSN 2056-9890

Received 3 February 2015

Accepted 26 February 2015

Edited by V. V. Chernyshev, Moscow State University, Russia

Keywords: crystal structure; bisphosphonate complexes; complexes; cadmium; powder diffraction; octahedral coordination; zwitterion; hydrogen bonding

CCDC reference: 1051338

Supporting information: this article has supporting information at journals.iucr.org/e

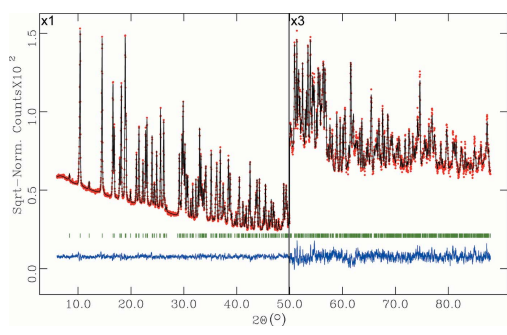

OPEN $\odot$ ACCESS

\section{Crystal structure of bis[(1-ammonio-1-phosphono- ethyl)phosphonato]tetraaquacadmium dihydrate: a powder X-ray diffraction study}

\author{
Mwaffak Rukiah $^{\mathrm{a} *}$ and Thaer Assaad ${ }^{\mathrm{b}}$ \\ a Department of Chemistry, Atomic Energy Commission of Syria (AECS), PO Box 6091, Damascus, Syrian Arab Republic, \\ and ${ }^{\mathbf{b}}$ Radioisotope Department, Atomic Energy Commission of Syria (AECS), PO Box 6091, Damascus, Syrian Arab \\ Republic. *Correspondence e-mail: cscientific@aec.org.sy
}

In the title compound, $\left[\mathrm{Cd} L_{2}\left(\mathrm{H}_{2} \mathrm{O}\right)_{4}\right] \cdot 2 \mathrm{H}_{2} \mathrm{O}[L=$ (1-ammonio-1-phosphonoethyl)phosphonate, $\mathrm{C}_{2} \mathrm{H}_{8} \mathrm{NO}_{6} \mathrm{P}_{2}^{-}$], the $\mathrm{Cd}^{\mathrm{II}}$ ion is situated on an inversion centre being coordinated by four aqua molecules in the equatorial plane and two phosphonate $\mathrm{O}$ atoms from two deprotonated $L$ ligands in the axial positions in a distorted octahedral geometry. The asymmetric unit contains one-half of the complex molecule and one lattice water molecule. The ligand $L$ exists in a zwitterionic form, with a positive charge on the $\mathrm{NH}_{3}$ group and a negative charge on the $\mathrm{O}$ atom of the non-coordinating phosphonate group, and with an intramolecular $\mathrm{O}-\mathrm{H} \cdots \mathrm{O}$ interaction forming an $S(6)$ ring motif and two intramolecular $\mathrm{N}-\mathrm{H} \cdots \mathrm{O}$ interactions each generating an $S(5)$ ring motif. In the crystal, $\mathrm{N}-\mathrm{H} \cdots \mathrm{O}$ and $\mathrm{O}-\mathrm{H} \cdots \mathrm{O}$ hydrogen bonds link the complex molecules into a three-dimensional network in which the voids of $38 \AA^{3}$ are filled with ordered lattice water molecules, which are also involved in $\mathrm{O}-\mathrm{H} \cdots \mathrm{O}$ hydrogen bonding.

\section{Chemical context}

As a result of of their inhibitory effect on bone resorption, various types of bisphosphonates are used in the treatment of bone metastasis and several bone disorders such as Paget's disease, and for the prevention of osteoporosis in postmenopausal women (Shaw \& Bishop, 2005). Drugs prepared on the basis of bisphosphonates are highly efficient as a regulator of calcium metabolism and the immune response; they are used as anti-neoplastic, anti-inflammatory and antiviral agents, drugs with analgesic effect and, as a component of toothpastes, biphosphonates prevent the formation of tartar (Matkovskaya et al., 2001). Organic diphosphonic acids are potentially very powerful chelating agents, used in metal extractions and have been tested by the pharmaceutical industry for use as efficient drugs preventing calcification and inhibiting bone resorption (Matczak-Jon \& VidenovaAdrabińska, 2005). Diphosphonic acids and their metal complexes are used in the treatment of Paget's disease, osteoporosis and tumoral osteolysis (Szabo et al., 2002). However, it is still not clearly understood why small structural modification of bisphosphonates may lead to extensive alterations in their physicochemical, biological and toxicological characteristics (Matczak-Jon \& Videnova-Adrabińska, 2005). Therefore, the structure determination of bisphosphonates is very important in order to understand the influence of structural modifications on their complex-forming abilities and physiological activities. 


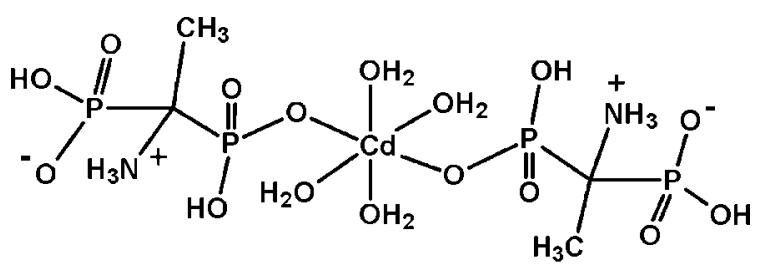

$2 \mathrm{H}_{2} \mathrm{O}$

\section{Structural commentary}

The asymmetric unit of the title compound, (I) (Fig. 1), contains one half of the complex molecule $\left[\mathrm{Cd} L_{2}\left(\mathrm{H}_{2} \mathrm{O}\right)_{4}\right][L=$ (1-ammonio-1-phosphonoethyl)phosphonate] and one lattice water molecule. All bond lengths and angles in (I) are normal and correspond to those observed in bisphosphonate complexes with transition metals (Shkol'nikova et al., 1991; Sergienko et al., 1997, 1999; Yin et al., 2005; Li et al., 2006; Li \& Sun, 2007; Lin et al., 2007; Xiang et al., 2007; Dudko et al., 2009, 2010; Bon et al., 2010; Tsaryk et al., 2010, 2011). The $\mathrm{Cd}^{\mathrm{II}}$ atom occupies a special position on an inversion centre and shows a slightly distorted octahedral coordination environment formed by two phosphonic $\mathrm{O}$ atoms in trans positions and four aqua $\mathrm{O}$ atoms in the equatorial plane. The distorted octahedral coordination polyhedron is slightly compressed in the axial direction; the $\mathrm{Cd} 1-\mathrm{O} 2$ bond length is $0.1 \AA$ shorter than the $\mathrm{Cd} 1-\mathrm{O} 1 W$ and $\mathrm{Cd} 1-\mathrm{O} 2 W$ bonds. The values of the axial $\mathrm{O}-\mathrm{Cd}-\mathrm{O}$ angles are in the range 80.1 (4)-99.9 (4) ${ }^{\circ}$, indicating a significant deviation from ideal values. The ligand $L$ exists in a zwitterionic form, with a positive charge on the $\mathrm{NH}_{3}$ group and a negative charge on the $\mathrm{O}$ atom of the non-coordinating phosphonate group, and with an intramolecular $\mathrm{O}-$ $\mathrm{H}$.. O interaction forming an $S(6)$ ring motif and two intramolecular $\mathrm{N}-\mathrm{H} \cdots \mathrm{O}$ interactions each generating an $S(5)$ ring motif (Table 1).

\section{Supramolecular features}

The crystal packing is illustrated in Fig. 2 as a projection of the unit cell along the $b$ axis. Intermolecular $\mathrm{N}-\mathrm{H} \cdots \mathrm{O}$ and $\mathrm{O}-$ $\mathrm{H}$... O hydrogen bonds (Table 1) link complex molecules into a three-dimensional network in which the voids of $38 \AA^{3}$ are

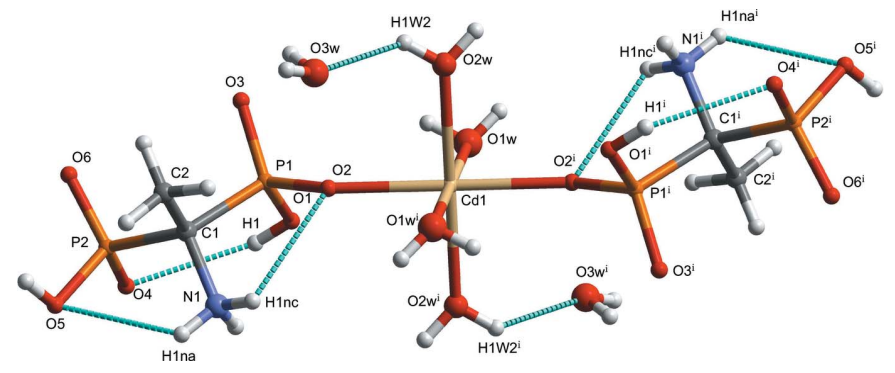

Figure 1

The molecular structure of (I), showing the atom-labelling scheme [symmetry code: (i) $-x+1,-y+1,-z+1$ ]. Displacement spheres are drawn at the $50 \%$ probability level. $\mathrm{H}$ atoms are represented as small spheres of arbitrary radii. Dotted lines denote hydrogen bonds.
Table 1

Hydrogen-bond geometry $\left(\AA,^{\circ}\right)$.

\begin{tabular}{|c|c|c|c|c|}
\hline$D-\mathrm{H} \cdots A$ & $D-\mathrm{H}$ & $\mathrm{H} \cdots A$ & $D \cdots A$ & $D-\mathrm{H} \cdots A$ \\
\hline $\mathrm{O} 1-\mathrm{H} 1 \cdots \mathrm{O} 4$ & 0.84 & 2.44 & $3.196(13)$ & 151 \\
\hline $\mathrm{N} 1-\mathrm{H} 1 \mathrm{NA} \cdots \mathrm{O}^{\mathrm{i}}$ & 0.87 & 2.07 & $2.828(13)$ & 146 \\
\hline $\mathrm{N} 1-\mathrm{H} 1 \mathrm{NB} \cdots \mathrm{O} 4^{\mathrm{ii}}$ & 0.88 & 2.16 & $2.872(15)$ & 137 \\
\hline $\mathrm{N} 1-\mathrm{H} 1 \mathrm{NC} \cdots \mathrm{O} 3^{\mathrm{i}}$ & 0.86 & 1.99 & $2.796(14)$ & 156 \\
\hline $\mathrm{O} 1 W-\mathrm{H} 1 W 1 \cdots \mathrm{O} 2 W^{\mathrm{i}}$ & 0.82 & 2.39 & $2.987(13)$ & 131 \\
\hline $\mathrm{O} 5-\mathrm{H} 5 \cdots \mathrm{O} 6^{\mathrm{iii}}$ & 0.84 & 1.79 & $2.551(12)$ & 150 \\
\hline $\mathrm{O} 1 W-\mathrm{H} 2 W 1 \cdots \mathrm{O} 3^{\mathrm{i}}$ & 0.82 & 1.96 & $2.758(15)$ & 162 \\
\hline $\mathrm{O} 2 W-\mathrm{H} 2 W 2 \cdots \mathrm{O} 4^{\mathrm{iv}}$ & 0.82 & 2.35 & $3.141(15)$ & 162 \\
\hline $\mathrm{O} 3 \mathrm{~W}-\mathrm{H} 1 W 3 \cdots \mathrm{O} 3 \mathrm{~W}^{\mathrm{v}}$ & 0.82 & 2.56 & $3.346(13)$ & 160 \\
\hline $\mathrm{O} 3 \mathrm{~W}-\mathrm{H} 2 W 3 \cdots \mathrm{O} 3^{\mathrm{vi}}$ & 0.82 & 2.13 & $2.833(14)$ & 143 \\
\hline
\end{tabular}

Symmetry codes: (i) $x, y+1, z$; (ii) $-x,-y+1,-z+1$; (iii) $-x, y+\frac{1}{2},-z+\frac{3}{2}$; (iv) $x+1, y, z ;(\mathrm{v})-x+1, y-\frac{1}{2},-z+\frac{3}{2} ;(\mathrm{vi})-x+1, y+\frac{1}{2},-z+\frac{3}{2}$.

filled with ordered lattice water molecules, which are also involved in $\mathrm{O}-\mathrm{H}$.. O hydrogen bonding (Table 1 and Fig. 2).

\section{Synthesis and crystallization}

All reactions and manipulations were carried out in air with reagent grade solvents. 1-Aminoethane-1,1-diyldiphosphonic acid was prepared according to the literature method of Rukiah \& Assaad (2013). The title compound (I) was prepared by adding $10 \mathrm{ml}$ of an $0.01 \mathrm{M} \mathrm{CdCl}_{2}$ aqueous solution to $10 \mathrm{ml}$ of a $0.02 \mathrm{M}$ water solution of 1-aminoethane-1,1diyldiphosphonic acid. A crude product was obtained after two weeks of slow evaporation of the resulted solution. It was further purified by recrystallization from ethanol and water $(1: 3 v / v)$ at $273 \mathrm{~K}$ to produce the title compound (I) (white powder; m.p. $>623 \mathrm{~K}$ ) in $80 \%$ yield. The IR spectrum was recorded on a Jasco FT-IR 300E instrument and the ${ }^{1} \mathrm{H}$ and ${ }^{13} \mathrm{C}\left\{{ }^{1} \mathrm{H}\right\}$ NMR spectra were recorded on a Bruker Bio spin 400 spectrometer.

\section{Spectroscopic data for (I):}

${ }^{1} \mathrm{H}$ NMR (D ${ }_{2} \mathrm{O}$, p.p.m.): $\delta 1.67\left(t, 3 \mathrm{H}, \mathrm{CH}_{3}, J=14 \mathrm{~Hz}\right)$. ${ }^{13} \mathrm{C}\left\{{ }^{1} \mathrm{H}\right\}$ NMR (D $2 \mathrm{O}$, p.p.m.): $\delta 20.5\left(1 \mathrm{C} ; \mathrm{CH}_{3}\right), 54.7(1 \mathrm{C} ; \mathrm{C}-$ $\left.\mathrm{CH}_{3}\right) .{ }^{31} \mathrm{P}\left\{{ }^{1} \mathrm{H}\right\}$ NMR $\left(\mathrm{D}_{2} \mathrm{O}\right.$, p.p.m.): $\delta$ 13.61(2P; P-OH). IR $\left(\mathrm{KBr}, v \mathrm{~cm}^{-1}\right): 3446.2\left(\mathrm{NH}_{3}\right), 2351.5(\mathrm{POH}), 1605.0(\mathrm{O}=\mathrm{P}-$ $\mathrm{O}-\mathrm{H})$.

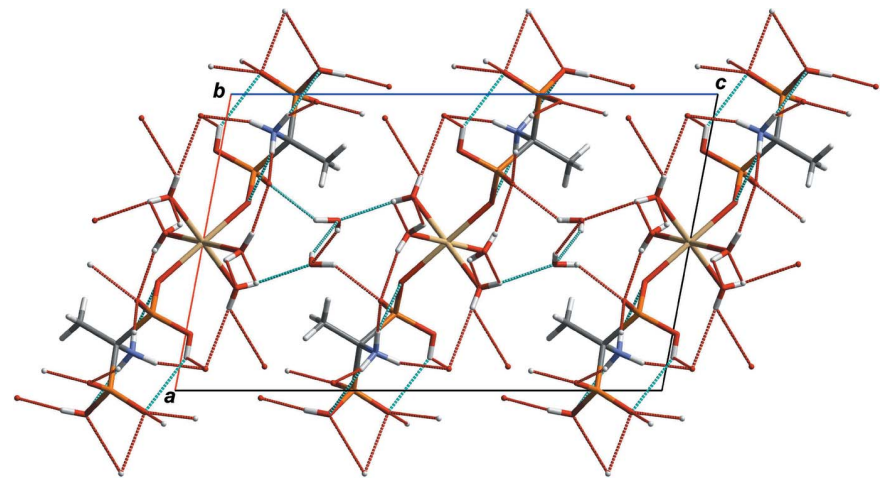

Figure 2

A portion of the crystal packing viewed down the $b$ axis. Dashed lines denote hydrogen bonds. 
Table 2

Experimental details.

\begin{tabular}{ll}
\hline Crystal data & {$\left[\mathrm{Cd}\left(\mathrm{C}_{2} \mathrm{H}_{8} \mathrm{NO}_{6} \mathrm{P}_{2}\right)_{2}\left(\mathrm{H}_{2} \mathrm{O}\right)_{4}\right] \cdot 2 \mathrm{H}_{2} \mathrm{O}$} \\
Chemical formula & 628.57 \\
$M_{\mathrm{r}}$ & Monoclinic, $P 2_{1} / c$ \\
Crystal system, space group & 298 \\
Temperature $(\mathrm{K})$ & $10.69424(12), 5.61453(5), 17.2737(2)$ \\
$a, b, c(\AA)$ & $100.7029(8)$ \\
$\beta\left({ }^{\circ}\right)$ & $1019.12(2)$ \\
$V\left(\AA^{3}\right)$ & 2 \\
$Z$ & Cu $K \alpha_{1}, \lambda=1.5406 \AA$ \\
Radiation type & 12.41 \\
$\mu\left(\mathrm{mm}^{-1}\right)$ & Flat sheet, $8 \times 8$ \\
Specimen shape, size $(\mathrm{mm})$ & \\
& \\
Data collection & Stoe transmission STADI-P \\
Diffractometer & Powder loaded into two Mylar foils \\
Specimen mounting & Transmission \\
Data collection mode & Step \\
Scan method & For a cylinder mounted on the $\varphi$ axis $[G S A S$ \\
Absorption correction & $($ Larson $\&$ Von Dreele, 2004) absorption/ \\
& surface roughness correction: function \\
& No. 4, flat-plate transmission absorption \\
& correction, terms $=0.75850]$ \\
$T_{\text {min }}, T_{\text {max }}$ & $0.195,0.310$ \\
$2 \theta$ values $\left(^{\circ}\right)$ & $2 \theta_{\text {min }}=6.002 \theta_{\text {max }}=89.98$ 2 $\theta_{\text {step }}=0.02$ \\
& \\
Refinement & \\
$R$ factors and goodness of fit & $R_{\mathrm{p}}=0.029, R_{\mathrm{wp}}=0.039, R_{\text {exp }}=0.025, R\left(F^{2}\right)$ \\
& $=0.04534, \chi^{2}=2.624$ \\
No. of data points & 4100 \\
No. of parameters & 133 \\
No. of restraints & 4 \\
H-atom treatment & H-atom parameters not refined \\
\hline &
\end{tabular}

Computer programs: WinXPOW (Stoe \& Cie, 1999), GSAS (Larson \& Von Dreele, 2004), EXPO2014 (Altomare et al., 2013), Mercury (Macrae et al., 2006) and publCIF (Westrip, 2010).

\section{Refinement}

Crystal data, data collection and structure refinement details are summarized in Table 2. Compound (I) has a tendency to crystallize in the form of a very fine white powder. Since no single crystals of sufficient size and quality could be obtained, a crystal structure determination from laboratory powder $\mathrm{X}$-ray diffraction data was performed. The powder sample was ground slightly in a mortar, loaded into two Mylar foils and fixed onto the sample holder with a mask of suitable internal diameter $(8.0 \mathrm{~mm})$. The powder X-ray diffraction data were collected at room temperature with a STOE transmission STADI-P diffractometer using $\mathrm{CuK}_{\alpha 1}$ radiation $(\lambda=$ $1.54060 \AA$ ) selected with an incident-beam curved-crystal $\mathrm{Ge}(111)$ monochromator with a linear position-sensitive detector (PSD). The pattern was scanned over the angular range $6.0-90.0^{\circ}(2 \theta)$. For pattern indexing, extraction of the peak positions was carried out with the program WinPLOTR (Roisnel \& Rodríguez-Carvajal, 2001). Pattern indexing was performed with the program DICVOL4.0 (Boultif \& Louër, 2004). The first 20 intense peaks of the powder pattern were indexed completely on the basis of a monoclinic cell. The figures of merit (de Wolff et al., 1968; Smith \& Snyder, 1979) are sufficiently acceptable to support the obtained indexing results $[M(20)=37.1, F(20)=78.5(0.0061,42)]$. The best estimated monoclinic space group was $P 2_{1} / c$.

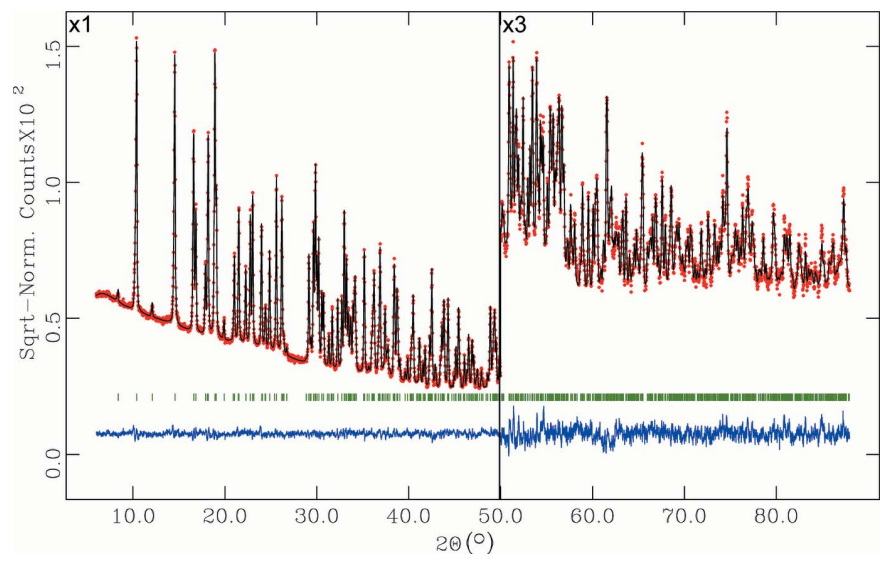

Figure 3

The final Rietveld plot for (I). Experimental intensities are indicated by dots, and the best-fit calculated (upper trace) and difference (lower trace) patterns are shown as solid lines. The vertical bars indicate the calculated positions of the Bragg peaks.

The powder pattern was subsequently refined with cell and resolution constraints (Le Bail et al., 1988) using the profilematching option of the program FULLPROF (RodríguezCarvajal, 2001). The number of molecules per unit cell was estimated to be $Z=2$. The initial crystal structure was determined by direct methods using the program EXPO2014 (Altomare et al., 2013). The model found by this program was introduced into the program GSAS (Larson \& Von Dreele, 2004) implemented in EXPGUI (Toby, 2001) for Rietveld refinement. The background was refined using a shifted Chebyshev polynomial with 20 coefficients. The effect of the asymmetry of the low-order peaks was corrected using a pseudo-Voigt description of the peak shape (Thompson et al., 1987), angle-dependent asymmetry with axial divergence (Finger et al., 1994) and microstrain broadening (Stephens, $1999)$. Two asymmetry parameters of this function, $S / L$ and $D /$ $L$, were both fixed at 0.0225 during this refinement. Intensities were corrected for absorption effects with a function for a plate sample in transmission geometry with $\mu \cdot d$ value of 0.7585 ( $\mu$ is the absorption coefficient and $d$ is the sample thickness). These $\mu \cdot d$ values were determined experimentally. The preferred orientation was modelled with 12 coefficients using a spherical harmonics correction (Von Dreele, 1997) of intensities. The use of the preferred orientation correction leads to a better molecular geometry with better agreement factors. The value of obtained median texture index (1.0654) and the agreement factors in the refinement without texture correction $\left(R_{\mathrm{p}}=0.053, R_{\mathrm{wp}}=0.073, R_{\exp }=0.025, R\left(F^{2}\right)=\right.$ 0.011009 and $\left.\chi^{2}=8.940\right)$ indicate that the preferred orientation improvement of the refinement is considerable.

Before the final refinement, the $\mathrm{H}$ atoms of the $\mathrm{CH}_{3}$ and $\mathrm{NH}_{3}$ groups were introduced on the basis of geometrical arguments. The hydroxy and water $\mathrm{H}$ atoms were located using the program HYDROGEN (Nardelli, 1999) implemented in Win $G X$ (Farrugia, 2012). The coordinates of all $\mathrm{H}$ atoms were refined with very strict soft restraints on bond lengths and angle until a suitable geometry was obtained, after 
that they were fixed in the final stage of the refinement. Four restraints for the central carbon atom $\left(\mathrm{C}-\mathrm{CH}_{3}, \mathrm{C}-\mathrm{NH}_{3}\right.$ and two $\mathrm{C}-\mathrm{PO}_{3}$ ) on bond lengths were applied to normal values for these bonds. The final refinement cycles were performed varying isotropic displacement parameters for $\mathrm{Cd}$ and water $\mathrm{O}$ atoms, and fixed isotropic displacement parameters for $\mathrm{P}, \mathrm{C}$, $\mathrm{N}, \mathrm{O}$ and $\mathrm{H}$ atoms. The final Rietveld plot is shown in Fig. 3.

\section{Acknowledgements}

We thank Professor I. Othman, Director General, Professor Z. Ajii, Head of the Chemistry Department, and Professor A. H. Al-Rayyes, Head of the Radioisotope Department, for their support of this work. We also thank Mr Emad Ghanem and Madame Najwa Karajoli for their kind assistance with the laboratory work.

\section{References}

Altomare, A., Cuocci, C., Giacovazzo, C., Moliterni, A., Rizzi, R., Corriero, N. \& Falcicchio, A. (2013). J. Appl. Cryst. 46, 1231-1235.

Bon, V. V., Dudko, A. V., Kozachkova, A. N., Pekhnyo, V. I. \& Tsaryk, N. V. (2010). Acta Cryst. E66, m537-m538.

Boultif, A. \& Louër, D. (2004). J. Appl. Cryst. 37, 724-731.

Dudko, A., Bon, V., Kozachkova, A. \& Pekhnyo, V. (2009). Acta Cryst. E65, m459.

Dudko, A., Bon, V., Kozachkova, A., Tsaryk, N. \& Pekhnyo, V. (2010). Acta Cryst. E66, m170-m171.

Farrugia, L. J. (2012). J. Appl. Cryst. 45, 849-854.

Finger, L. W., Cox, D. E. \& Jephcoat, A. P. (1994). J. Appl. Cryst. 27, 892-900.

Larson, A. C. \& Von Dreele, R. B. (2004). GSAS. Report LAUR 86748. Los Alamos National Laboratory, New Mexico, USA.

Le Bail, A., Duroy, H. \& Fourquet, J. L. (1988). Mater. Res. Bull. 23, 447-452.

Li, M., Chen, S., Xiang, J., He, H., Yuan, L. \& Sun, J. (2006). Cryst. Growth Des. 6, 1250-1252.

Li, M. \& Sun, J.-T. (2007). Acta Cryst. E63, m1370-m1372.
Lin, L., Zhang, T., Fan, Y., Ding, D. \& Hou, H. (2007). J. Mol. Struct. 837, 107-117.

Macrae, C. F., Edgington, P. R., McCabe, P., Pidcock, E., Shields, G. P., Taylor, R., Towler, M. \& van de Streek, J. (2006). J. Appl. Cryst. 39, 453-457.

Matczak-Jon, E. \& Videnova-Adrabińska, V. (2005). Coord. Chem. Rev. 249, 2458-2488.

Matkovskaya, T. A., Popov, K. I. \& Yuryeva, E. A. (2001). Bisphosphonates. Properties, Structure and Application in Medicine, p. 223. Moscow: Khimiya.

Nardelli, M. (1999). J. Appl. Cryst. 32, 563-571.

Rodríguez-Carvajal, J. (2001). Commission on Powder Diffraction (IUCr) Newsletter, 26, 12-19.

Roisnel, T. \& Rodríguez-Carvajal, J. (2001). Mater. Sci. Forum, 378381, 118-123.

Rukiah, M. \& Assaad, T. (2013). Acta Cryst. C69, 815-818.

Sergienko, V. S., Afonin, E. G. \& Aleksandrov, G. G. (1999). Koord. Khim. 25, 133-142.

Sergienko, V. S., Aleksandrov, G. G. \& Afonin, E. G. (1997). Zh. Neorg. Khim. 42, 1291-1296.

Shaw, N. J. \& Bishop, N. J. (2005). Arch. Dis. Child. 90, 494-499.

Shkol'nikova, L. M., Porai-Koshits, M. A., Fundamenskii, V. S., Poznyak, A. L. \& Kalugina, E. V. (1991). Koord. Khim. 17, 954-963.

Smith, G. S. \& Snyder, R. L. (1979). J. Appl. Cryst. 12, 60-65.

Stephens, P. W. (1999). J. Appl. Cryst. 32, 281-289.

Stoe \& Cie (1999). WinXPOW. Stoe \& Cie, Darmstadt, Germany.

Szabo, Ch. M., Martin, M. B. \& Oldfield, E. (2002). J. Med. Chem. 45, 2894-2903.

Thompson, P., Cox, D. E. \& Hastings, J. B. (1987). J. Appl. Cryst. 20, 79-83.

Toby, B. H. (2001). J. Appl. Cryst. 34, 210-213.

Tsaryk, N. V., Dudko, A. V., Kozachkova, A. N., Bon, V. V. \& Pekhnyo, V. I. (2010). Acta Cryst. E66, m1533-m1534.

Tsaryk, N. V., Dudko, A. V., Kozachkova, A. N. \& Pekhnyo, V. I. (2011). Acta Cryst. E67, o1651-o1652.

Von Dreele, R. B. (1997). J. Appl. Cryst. 30, 517-525.

Westrip, S. P. (2010). J. Appl. Cryst. 43, 920-925.

Wolff, P. M. de (1968). J. Appl. Cryst. 1, 108-113.

Xiang, J., Li, M., Wu, S., Yuan, L.-J. \& Sun, J. (2007). J. Mol. Struct. 826, 143-149.

Yin, P., Wang, X.-C., Gao, S. \& Zheng, L.-M. (2005). J. Solid State Chem. 178, 1049-1053. 


\section{supporting information}

Acta Cryst. (2015). E71, 342-345 [doi:10.1107/S2056989015004028]

\section{Crystal structure of bis[(1-ammonio-1-phosphonoethyl) phosphonato]tetraaqua-}

\section{cadmium dihydrate: a powder $\mathrm{X}$-ray diffraction study}

\section{Mwaffak Rukiah and Thaer Assaad}

\section{Computing details}

Data collection: WinXPOW (Stoe \& Cie, 1999); cell refinement: GSAS (Larson \& Von Dreele, 2004); data reduction:

WinXPOW (Stoe \& Cie, 1999); program(s) used to solve structure: EXPO2014 (Altomare et al., 2013); program(s) used to refine structure: GSAS (Larson \& Von Dreele, 2004); molecular graphics: Mercury (Macrae et al., 2006); software used to prepare material for publication: publCIF (Westrip, 2010).

Bis[(1-ammonio-1-phosphonoethyl) phosphonato]tetraaquacadmium dihydrate

\section{Crystal data}

$\left[\mathrm{Cd}\left(\mathrm{C}_{2} \mathrm{H}_{8} \mathrm{NO}_{6} \mathrm{P}_{2}\right)_{2}\left(\mathrm{H}_{2} \mathrm{O}\right)_{4}\right] \cdot 2 \mathrm{H}_{2} \mathrm{O}$

$M_{r}=628.57$

Monoclinic, $P 2{ }_{1} / c$

Hall symbol: -P $2 \mathrm{ybc}$

$a=10.69424(12) \AA$

$b=5.61453(5) \AA$

$c=17.2737(2) \AA$

$\beta=100.7029(8)^{\circ}$

$V=1019.12(2) \AA^{3}$

\section{Data collection}

Stoe transmission STADI-P diffractometer

Radiation source: sealed X-ray tube

Ge 111 monochromator

Specimen mounting: Powder loaded into two

Mylar foils

Data collection mode: transmission

Scan method: step

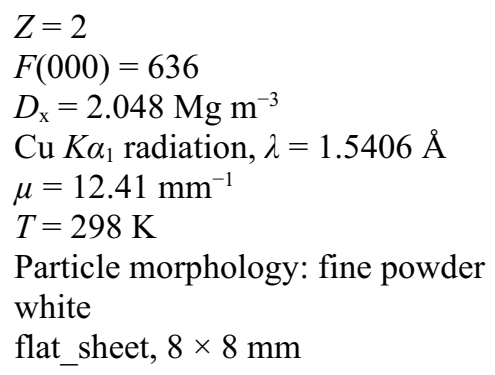

\footnotetext{
Absorption correction: for a cylinder mounted on the $\varphi$ axis [GSAS (Larson \& Von Dreele, 2004) absorption/surface roughness correction: function No. 4, flat-plate transmission absorption correction, terms $=0.75850]$ $T_{\min }=0.195, T_{\max }=0.310$

$2 \theta_{\min }=6.00^{\circ}, 2 \theta_{\max }=89.98^{\circ}, 2 \theta_{\text {step }}=0.02^{\circ}$
} 


\section{Refinement}

Refinement on $I_{\text {net }}$

Least-squares matrix: full

$R_{\mathrm{p}}=0.029$

$R_{\text {wp }}=0.039$

$R_{\text {exp }}=0.025$

$R\left(F^{2}\right)=0.04534$

4100 data points
Profile function: CW Profile function number 4 with 21 terms Pseudovoigt profile coefficients as parameterized in (Thompson et al., 1987). Asymmetry correction of (Finger et al., 1994). Microstrain broadening by (Stephens, 1999). $\# 1(\mathrm{GU})=0.000 \# 2(\mathrm{GV})=0.000 \# 3(\mathrm{GW})=$ $7.136 \# 4(\mathrm{GP})=0.000 \# 5(\mathrm{LX})=2.421 \# 6(\mathrm{ptec})$ $=0.11 \# 7$ (trns) $=0.00 \# 8(\mathrm{shft})=0.0000$ $\# 9(\mathrm{sfec})=0.00 \# 10(\mathrm{~S} / \mathrm{L})=0.0225 \# 11(\mathrm{H} / \mathrm{L})=$ $0.0225 \# 12$ (eta) $=0.6026 \# 13(\mathrm{~S} 400)=1.2 \mathrm{E}-02$ $\# 14(\mathrm{~S} 040)=1.0 \mathrm{E}-01 \# 15(\mathrm{~S} 004)=1.2 \mathrm{E}-03$ $\# 16(\mathrm{~S} 220)=3.3 \mathrm{E}-02 \# 17(\mathrm{~S} 202)=6.7 \mathrm{E}-03$ $\# 18(\mathrm{~S} 022)=1.7 \mathrm{E}-02 \# 19(\mathrm{~S} 301)=1.1 \mathrm{E}-02$ $\# 20(\mathrm{~S} 103)=2.3 \mathrm{E}-03 \# 21(\mathrm{~S} 121)=1.5 \mathrm{E}-02$ Peak tails are ignored where the intensity is below 0.0010 times the peak Aniso. broadening axis 0.00 .01 .0

133 parameters

4 restraints

$\mathrm{H}$-atom parameters not refined

Weighting scheme based on measured s.u.'s $(\Delta / \sigma)_{\max }=0.03$

Background function: GSAS Background function number 1 with 20 terms. Shifted Chebyshev function of 1st kind 1: 1216.00 2: -1325.95 3: 695.908 4: -224.478 5: 51.5854 6: -12.9254 7: 7.98937 8: -13.4593 9: 4.35490 10: 32.6578 11: -32.8988 12: -6.52632 13: -18.8133 14: 23.4504 15: -2.70081 16: -0.874623 17: -28.0163 18: 27.3423 19: 2.28961 20: -11.2631

Fractional atomic coordinates and isotropic or equivalent isotropic displacement parameters $\left(\AA^{2}\right)$

\begin{tabular}{lllll}
\hline & $x$ & $y$ & $z$ & $U_{\text {iso }} / U_{\mathrm{eq}}$ \\
\hline $\mathrm{C} 1$ & $0.1555(5)$ & $0.3764(12)$ & $0.6381(5)$ & $0.05^{*}$ \\
$\mathrm{C} 2$ & $0.2285(11)$ & $0.4133(17)$ & $0.7222(5)$ & $0.015^{*}$ \\
$\mathrm{H} 2 \mathrm{a}$ & 0.17609 & 0.50488 & 0.75213 & $0.02^{*}$ \\
$\mathrm{H} 2 \mathrm{~b}$ & 0.3063 & 0.50177 & 0.72104 & $0.02^{*}$ \\
$\mathrm{H} 2 \mathrm{c}$ & 0.24919 & 0.26144 & 0.74755 & $0.02^{*}$ \\
$\mathrm{~N} 1$ & $0.1274(11)$ & $0.6170(16)$ & $0.6028(7)$ & $0.02^{*}$ \\
$\mathrm{H} 1 \mathrm{na}$ & 0.07228 & 0.68814 & 0.62561 & $0.025^{*}$ \\
$\mathrm{H} 1 \mathrm{nb}$ & 0.09382 & 0.59823 & 0.55236 & $0.025^{*}$ \\
$\mathrm{H} 1 \mathrm{nc}$ & 0.19624 & 0.69829 & 0.60678 & $0.025^{*}$ \\
P1 & $0.2594(5)$ & $0.2361(7)$ & $0.5774(3)$ & $0.01^{*}$ \\
O1 & $0.1897(8)$ & $0.2047(13)$ & $0.4907(6)$ & $0.01^{*}$ \\
$\mathrm{H} 1$ & 0.11151 & 0.1887 & 0.48952 & $0.015^{*}$ \\
O2 & $0.3659(9)$ & $0.4155(15)$ & $0.5771(6)$ & $0.01^{*}$ \\
O3 & $0.3024(8)$ & $-0.0094(16)$ & $0.6124(6)$ & $0.01^{*}$ \\
P2 & $0.0019(4)$ & $0.2252(6)$ & $0.6342(3)$ & $0.01^{*}$ \\
O4 & $-0.0728(9)$ & $0.1904(14)$ & $0.5539(6)$ & $0.01^{*}$ \\
O5 & $-0.0807(8)$ & $0.4058(11)$ & $0.6745(5)$ & $0.01^{*}$
\end{tabular}




\begin{tabular}{lllll} 
H5 & -0.06622 & 0.38276 & 0.7235 & $0.03^{*}$ \\
O6 & $0.0255(7)$ & $-0.0022(16)$ & $0.6785(5)$ & $0.01^{*}$ \\
Cd1 & 0.5 & 0.5 & 0.5 & $0.0093(7)^{*}$ \\
O1W & $0.5302(11)$ & $0.8211(15)$ & $0.5815(7)$ & $0.029(4)^{*}$ \\
H1W1 & 0.56 & 0.91732 & 0.55443 & $0.03^{*}$ \\
H2W1 & 0.47216 & 0.89419 & 0.59649 & $0.03^{*}$ \\
O2W & $0.6537(10)$ & $0.2995(14)$ & $0.5866(6)$ & $0.020(3)^{*}$ \\
H1W2 & 0.63758 & 0.22791 & 0.62489 & $0.03^{*}$ \\
H2W2 & 0.72024 & 0.23865 & 0.57871 & $0.03^{*}$ \\
O3w & $0.5739(11)$ & $0.3949(14)$ & $0.7310(6)$ & $0.028(3)^{*}$ \\
H1W3 & 0.54032 & 0.26314 & 0.72841 & $0.03^{*}$ \\
H2W3 & 0.5752 & 0.43812 & 0.77663 & $0.03^{*}$ \\
\hline
\end{tabular}

Geometric parameters $\left(\AA,^{\circ}\right)$

\begin{tabular}{|c|c|c|c|}
\hline $\mathrm{C} 1-\mathrm{C} 2$ & $1.530(12)$ & $\mathrm{P} 2-\mathrm{O} 5$ & $1.588(7)$ \\
\hline $\mathrm{C} 1-\mathrm{N} 1$ & $1.490(12)$ & $\mathrm{P} 2-\mathrm{O} 6$ & $1.486(9)$ \\
\hline $\mathrm{C} 1-\mathrm{P} 1$ & $1.840(9)$ & $\mathrm{O} 5-\mathrm{H} 5$ & 0.842 \\
\hline $\mathrm{C} 1-\mathrm{P} 2$ & $1.839(7)$ & $\mathrm{Cd} 1-\mathrm{O} 2$ & $2.183(8)$ \\
\hline $\mathrm{C} 2-\mathrm{H} 2 \mathrm{a}$ & 0.976 & $\mathrm{Cd} 1-\mathrm{O} 2^{\mathrm{i}}$ & $2.183(8)$ \\
\hline $\mathrm{C} 2-\mathrm{H} 2 \mathrm{~b}$ & 0.972 & $\mathrm{Cd} 1-\mathrm{O} 1 \mathrm{~W}$ & $2.274(9)$ \\
\hline $\mathrm{C} 2-\mathrm{H} 2 \mathrm{c}$ & 0.965 & $\mathrm{Cd} 1-\mathrm{O} 1 \mathrm{~W}^{\mathrm{i}}$ & $2.274(9)$ \\
\hline N1-H1na & 0.865 & $\mathrm{Cd} 1-\mathrm{O} 2 \mathrm{~W}$ & $2.300(10)$ \\
\hline $\mathrm{N} 1-\mathrm{H} 1 \mathrm{nb}$ & 0.885 & $\mathrm{Cd} 1-\mathrm{O} 2 \mathrm{~W}^{\mathrm{i}}$ & $2.300(10)$ \\
\hline $\mathrm{N} 1-\mathrm{H} 1 \mathrm{nc}$ & 0.858 & $\mathrm{O} 1 \mathrm{~W}-\mathrm{H} 1 \mathrm{~W} 1$ & 0.817 \\
\hline $\mathrm{P} 1-\mathrm{O} 1$ & $1.555(11)$ & $\mathrm{O} 1 \mathrm{~W}-\mathrm{H} 2 \mathrm{~W} 1$ & 0.824 \\
\hline $\mathrm{P} 1-\mathrm{O} 2$ & $1.521(9)$ & $\mathrm{O} 2 \mathrm{~W}-\mathrm{H} 1 \mathrm{~W} 2$ & 0.820 \\
\hline $\mathrm{P} 1-\mathrm{O} 3$ & $1.541(9)$ & $\mathrm{O} 2 \mathrm{~W}-\mathrm{H} 2 \mathrm{~W} 2$ & 0.823 \\
\hline $\mathrm{O} 1-\mathrm{H} 1$ & 0.838 & $\mathrm{O} 3 \mathrm{w}-\mathrm{H} 1 \mathrm{~W} 3$ & 0.820 \\
\hline $\mathrm{P} 2-\mathrm{O} 4$ & $1.480(10)$ & $\mathrm{O} 3 \mathrm{w}-\mathrm{H} 2 \mathrm{~W} 3$ & 0.823 \\
\hline $\mathrm{C} 2-\mathrm{C} 1-\mathrm{N} 1$ & $107.2(7)$ & $\mathrm{C} 1-\mathrm{P} 2-\mathrm{O} 6$ & $108.2(5)$ \\
\hline $\mathrm{C} 2-\mathrm{C} 1-\mathrm{P} 1$ & $110.1(6)$ & $\mathrm{O} 4-\mathrm{P} 2-\mathrm{O} 5$ & $104.3(5)$ \\
\hline $\mathrm{C} 2-\mathrm{C} 1-\mathrm{P} 2$ & $113.1(7)$ & $\mathrm{O} 4-\mathrm{P} 2-\mathrm{O} 6$ & $112.3(6)$ \\
\hline $\mathrm{N} 1-\mathrm{C} 1-\mathrm{P} 1$ & $104.6(5)$ & $\mathrm{O} 5-\mathrm{P} 2-\mathrm{O} 6$ & $112.2(5)$ \\
\hline $\mathrm{N} 1-\mathrm{C} 1-\mathrm{P} 2$ & $107.0(6)$ & $\mathrm{P} 2-\mathrm{O} 5-\mathrm{H} 5$ & 109.2 \\
\hline $\mathrm{P} 1-\mathrm{C} 1-\mathrm{P} 2$ & $114.3(4)$ & $\mathrm{O} 2-\mathrm{Cd} 1-\mathrm{O} 2^{\mathrm{i}}$ & 180.0 \\
\hline $\mathrm{C} 1-\mathrm{C} 2-\mathrm{H} 2 \mathrm{a}$ & 109.4 & $\mathrm{O} 2-\mathrm{Cd} 1-\mathrm{O} 1 \mathrm{~W}$ & $80.1(4)$ \\
\hline $\mathrm{C} 1-\mathrm{C} 2-\mathrm{H} 2 \mathrm{~b}$ & 109.6 & $\mathrm{O} 2-\mathrm{Cd} 1-\mathrm{O} 1 \mathrm{~W}^{\mathrm{i}}$ & $99.9(4)$ \\
\hline $\mathrm{C} 1-\mathrm{C} 2-\mathrm{H} 2 \mathrm{c}$ & 110.1 & $\mathrm{O} 2-\mathrm{Cd} 1-\mathrm{O} 2 \mathrm{~W}$ & $88.2(3)$ \\
\hline $\mathrm{H} 2 \mathrm{a}-\mathrm{C} 2-\mathrm{H} 2 \mathrm{~b}$ & 108.6 & $\mathrm{O} 2-\mathrm{Cd} 1-\mathrm{O} 2 \mathrm{~W}^{\mathrm{i}}$ & $91.8(3)$ \\
\hline $\mathrm{H} 2 \mathrm{a}-\mathrm{C} 2-\mathrm{H} 2 \mathrm{c}$ & 109.4 & $\mathrm{O} 2^{\mathrm{i}}-\mathrm{Cd} 1-\mathrm{O} 1 \mathrm{~W}$ & $99.9(4)$ \\
\hline $\mathrm{H} 2 \mathrm{~b}-\mathrm{C} 2-\mathrm{H} 2 \mathrm{c}$ & 109.7 & $\mathrm{O} 2^{\mathrm{i}}-\mathrm{Cd} 1-\mathrm{O} 1 \mathrm{~W}^{\mathrm{i}}$ & $80.1(4)$ \\
\hline $\mathrm{C} 1-\mathrm{N} 1-\mathrm{H} 1 \mathrm{na}$ & 109.5 & $\mathrm{O} 22^{\mathrm{i}}-\mathrm{Cd} 1-\mathrm{O} 2 \mathrm{~W}$ & $91.8(3)$ \\
\hline $\mathrm{C} 1-\mathrm{N} 1-\mathrm{H} 1 \mathrm{nb}$ & 108.0 & $\mathrm{O} 2^{\mathrm{i}}-\mathrm{Cd} 1-\mathrm{O} 2 \mathrm{~W}^{\mathrm{i}}$ & $88.2(3)$ \\
\hline $\mathrm{C} 1-\mathrm{N} 1-\mathrm{H} 1 \mathrm{nc}$ & 110.1 & $\mathrm{O} 1 \mathrm{~W}-\mathrm{Cd} 1-\mathrm{O} 1 \mathrm{~W}^{\mathrm{i}}$ & 180.0 \\
\hline $\mathrm{H} 1 \mathrm{na}-\mathrm{N} 1-\mathrm{H} 1 \mathrm{nb}$ & 108.5 & $\mathrm{O} 1 \mathrm{~W}-\mathrm{Cd} 1-\mathrm{O} 2 \mathrm{~W}$ & $89.0(4)$ \\
\hline $\mathrm{H} 1 \mathrm{na}-\mathrm{N} 1-\mathrm{H} 1 \mathrm{nc}$ & 111.4 & $\mathrm{O} 1 \mathrm{~W}-\mathrm{Cd} 1-\mathrm{O} 2 \mathrm{~W}^{\mathrm{i}}$ & $91.0(4)$ \\
\hline
\end{tabular}




\begin{tabular}{|c|c|c|c|}
\hline $\mathrm{H} 1 \mathrm{nb}-\mathrm{N} 1-\mathrm{H} 1 \mathrm{nc}$ & 109.1 & $\mathrm{O} 1 \mathrm{~W}^{\mathrm{i}}-\mathrm{Cd} 1-\mathrm{O} 2 \mathrm{~W}$ & $91.0(4)$ \\
\hline $\mathrm{C} 1-\mathrm{P} 1-\mathrm{O} 1$ & $111.5(6)$ & $\mathrm{O} 1 \mathrm{~W}^{\mathrm{i}}-\mathrm{Cd} 1-\mathrm{O} 2 \mathrm{~W}^{\mathrm{i}}$ & $89.0(4)$ \\
\hline $\mathrm{C} 1-\mathrm{P} 1-\mathrm{O} 2$ & $104.5(5)$ & $\mathrm{O} 2 \mathrm{~W}-\mathrm{Cd} 1-\mathrm{O} 2 \mathrm{~W}^{\mathrm{i}}$ & 180.0 \\
\hline $\mathrm{C} 1-\mathrm{P} 1-\mathrm{O} 3$ & $109.1(4)$ & $\mathrm{Cd} 1-\mathrm{O} 1 \mathrm{~W}-\mathrm{H} 1 \mathrm{~W} 1$ & 101.2 \\
\hline $\mathrm{O} 1-\mathrm{P} 1-\mathrm{O} 2$ & $107.2(5)$ & $\mathrm{Cd} 1-\mathrm{O} 1 \mathrm{~W}-\mathrm{H} 2 \mathrm{~W} 1$ & 124.1 \\
\hline $\mathrm{O} 1-\mathrm{P} 1-\mathrm{O} 3$ & $109.4(5)$ & $\mathrm{H} 1 \mathrm{~W} 1-\mathrm{O} 1 \mathrm{~W}-\mathrm{H} 2 \mathrm{~W} 1$ & 104.3 \\
\hline $\mathrm{O} 2-\mathrm{P} 1-\mathrm{O} 3$ & $115.1(6)$ & $\mathrm{Cd} 1-\mathrm{O} 2 \mathrm{~W}-\mathrm{H} 1 \mathrm{~W} 2$ & 122.2 \\
\hline $\mathrm{P} 1-\mathrm{O} 1-\mathrm{H} 1$ & 109.4 & $\mathrm{Cd} 1-\mathrm{O} 2 \mathrm{~W}-\mathrm{H} 2 \mathrm{~W} 2$ & 129.1 \\
\hline $\mathrm{P} 1-\mathrm{O} 2-\mathrm{Cd} 1$ & $136.4(6)$ & $\mathrm{H} 1 \mathrm{~W} 2-\mathrm{O} 2 \mathrm{~W}-\mathrm{H} 2 \mathrm{~W} 2$ & 104.3 \\
\hline $\mathrm{C} 1-\mathrm{P} 2-\mathrm{O} 4$ & $114.8(5)$ & $\mathrm{H} 1 \mathrm{~W} 3-\mathrm{O} 3 \mathrm{w}-\mathrm{H} 2 \mathrm{~W} 3$ & 104.3 \\
\hline $\mathrm{C} 1-\mathrm{P} 2-\mathrm{O} 5$ & $104.8(4)$ & & \\
\hline $\mathrm{O} 1 \mathrm{~W}-\mathrm{Cd} 1-\mathrm{O} 2-\mathrm{P} 1$ & $168.8(9)$ & $\mathrm{O} 1-\mathrm{P} 1-\mathrm{C} 1-\mathrm{C} 2$ & $-177.9(6)$ \\
\hline $\mathrm{O} 2 \mathrm{~W}-\mathrm{Cd} 1-\mathrm{O} 2-\mathrm{P} 1$ & $-101.9(8)$ & $\mathrm{O} 2-\mathrm{P} 1-\mathrm{C} 1-\mathrm{C} 2$ & $-62.3(7)$ \\
\hline $\mathrm{O} 1 \mathrm{~W}^{\mathrm{i}}-\mathrm{Cd} 1-\mathrm{O} 2-\mathrm{P} 1$ & $-11.2(9)$ & $\mathrm{O} 3-\mathrm{P} 1-\mathrm{C} 1-\mathrm{C} 2$ & $61.3(7)$ \\
\hline $\mathrm{O} 2 \mathrm{~W}^{\mathrm{i}}-\mathrm{Cd} 1-\mathrm{O} 2-\mathrm{P} 1$ & $78.1(8)$ & $\mathrm{O} 4-\mathrm{P} 2-\mathrm{C} 1-\mathrm{P} 1$ & $-54.2(6)$ \\
\hline $\mathrm{O} 3-\mathrm{P} 1-\mathrm{O} 2-\mathrm{Cd} 1$ & $84.9(9)$ & $\mathrm{O} 5-\mathrm{P} 2-\mathrm{C} 1-\mathrm{P} 1$ & $-168.0(5)$ \\
\hline $\mathrm{C} 1-\mathrm{P} 1-\mathrm{O} 2-\mathrm{Cd} 1$ & $-155.4(7)$ & $\mathrm{O} 6-\mathrm{P} 2-\mathrm{C} 1-\mathrm{P} 1$ & $72.1(6)$ \\
\hline $\mathrm{O} 1-\mathrm{P} 1-\mathrm{O} 2-\mathrm{Cd} 1$ & $-37.0(9)$ & $\mathrm{O} 4-\mathrm{P} 2-\mathrm{C} 1-\mathrm{N} 1$ & $61.1(8)$ \\
\hline $\mathrm{O} 1-\mathrm{P} 1-\mathrm{C} 1-\mathrm{P} 2$ & $53.6(6)$ & $\mathrm{O} 5-\mathrm{P} 2-\mathrm{C} 1-\mathrm{N} 1$ & $-52.7(8)$ \\
\hline $\mathrm{O} 2-\mathrm{P} 1-\mathrm{C} 1-\mathrm{P} 2$ & $169.2(5)$ & $\mathrm{O} 6-\mathrm{P} 2-\mathrm{C} 1-\mathrm{N} 1$ & $-172.6(7)$ \\
\hline $\mathrm{O} 3-\mathrm{P} 1-\mathrm{C} 1-\mathrm{P} 2$ & $-67.2(6)$ & $\mathrm{O} 4-\mathrm{P} 2-\mathrm{C} 1-\mathrm{C} 2$ & $178.9(6)$ \\
\hline $\mathrm{O} 1-\mathrm{P} 1-\mathrm{C} 1-\mathrm{N} 1$ & $-63.1(7)$ & $\mathrm{O} 5-\mathrm{P} 2-\mathrm{C} 1-\mathrm{C} 2$ & $65.0(7)$ \\
\hline $\mathrm{O} 2-\mathrm{P} 1-\mathrm{C} 1-\mathrm{N} 1$ & $52.5(8)$ & $\mathrm{O} 6-\mathrm{P} 2-\mathrm{C} 1-\mathrm{C} 2$ & $-54.9(7)$ \\
\hline $\mathrm{O} 3-\mathrm{P} 1-\mathrm{C} 1-\mathrm{N} 1$ & $176.1(7)$ & & \\
\hline
\end{tabular}

Symmetry code: (i) $-x+1,-y+1,-z+1$.

Hydrogen-bond geometry $\left(A,{ }^{\circ}\right)$

\begin{tabular}{|c|c|c|c|c|}
\hline$D-\mathrm{H} \cdots A$ & $D-\mathrm{H}$ & $\mathrm{H} \cdots A$ & $D \cdots A$ & $D-\mathrm{H} \cdots A$ \\
\hline $\mathrm{O} 1-\mathrm{H} 1 \cdots \mathrm{O} 4$ & 0.84 & 2.44 & $3.196(13)$ & 151 \\
\hline $\mathrm{O} 1-\mathrm{H} 1 \cdots \mathrm{O} 4^{\mathrm{ii}}$ & 0.84 & 2.27 & $2.592(12)$ & 103 \\
\hline 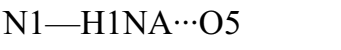 & 0.87 & 2.53 & $2.986(14)$ & 114 \\
\hline N1-H1NA $\cdots$ O $^{\mathrm{iii}}$ & 0.87 & 2.07 & $2.828(13)$ & 146 \\
\hline $\mathrm{N} 1-\mathrm{H} 1 \mathrm{NB} \cdots \mathrm{O} 4^{\mathrm{iv}}$ & 0.88 & 2.16 & $2.872(15)$ & 137 \\
\hline $\mathrm{N} 1-\mathrm{H} 1 \mathrm{NC} \cdots \mathrm{O} 2$ & 0.86 & 2.53 & $2.899(15)$ & 107 \\
\hline $\mathrm{N} 1-\mathrm{H} 1 \mathrm{NC} \cdots \mathrm{O} 3^{\mathrm{iii}}$ & 0.86 & 1.99 & $2.796(14)$ & 156 \\
\hline $\mathrm{O} 1 W-\mathrm{H} 1 W 1 \cdots \mathrm{O} 2 W^{\text {iii }}$ & 0.82 & 2.39 & $2.987(13)$ & 131 \\
\hline $\mathrm{O} 5-\mathrm{H} 5 \cdots \mathrm{O}^{v}$ & 0.84 & 1.79 & $2.551(12)$ & 150 \\
\hline $\mathrm{O} 1 W-\mathrm{H} 2 W 1 \cdots \mathrm{O} 3^{\mathrm{iii}}$ & 0.82 & 1.96 & $2.758(15)$ & 162 \\
\hline $\mathrm{O} 2 W-\mathrm{H} 1 W 2 \cdots \mathrm{O} 3 \mathrm{~W}$ & 0.82 & 2.27 & $2.833(15)$ & 126 \\
\hline $\mathrm{O} 2 W-\mathrm{H} 2 W 2 \cdots \mathrm{O} 4^{\mathrm{vi}}$ & 0.82 & 2.35 & $3.141(15)$ & 162 \\
\hline $\mathrm{O} 3 \mathrm{~W}-\mathrm{H} 1 W 3 \cdots \mathrm{O} 3 \mathrm{~W}^{\mathrm{vii}}$ & 0.82 & 2.56 & $3.346(13)$ & 160 \\
\hline $\mathrm{O} 3 \mathrm{~W}-\mathrm{H} 2 W 3 \cdots \mathrm{O} 3^{\text {viii }}$ & 0.82 & 2.13 & $2.833(14)$ & 143 \\
\hline
\end{tabular}

Symmetry codes: (ii) $-x,-y,-z+1$; (iii) $x, y+1, z$; (iv) $-x,-y+1,-z+1$; (v) $-x, y+1 / 2,-z+3 / 2$; (vi) $x+1, y, z$; (vii) $-x+1, y-1 / 2,-z+3 / 2$; (viii) $-x+1, y+1 / 2$, $-z+3 / 2$. 